\title{
3D Ultrasound real-time monitoring of surgical tools
}

\author{
Fabian Gaufillet ${ }^{1}$, Hervé Liegbott ${ }^{1}$, Marián Uherčík ${ }^{1,2}$, Fréderic Cervenansky ${ }^{1}$, Jan Kybic $^{2}$, Christian Cachard $^{1}$ \\ ${ }^{1}$ CREATIS-LRMN, Université de Lyon, INSA-Lyon, Université Lyon 1, CNRS UMR 5520, Inserm U630, \\ Villeurbanne, France \\ ${ }^{2}$ Center for Machine Perception, Department of Cybernetics, Faculty of Electrical Engineering, \\ Czech Technical University, Prague, Czech Republic
}

\begin{abstract}
To assist surgeons during their surgical operations, which involve a tool insertion, a real-time application which is able to localise the surgical tool during its movement is proposed. The position of the needle is estimated with a method based on model fitting using a Random Sample Consensus (RANSAC). Our proposed application has been implemented on the ULTRASONIX RP scanner and it permits 3D volumes acquisition, storage and the tool localization. The developed graphical user interface shows both the section plane containing the tool and the perpendicular plane to it in order to give a view of surrounding tissue.

The performed experiments have shown that our application is able to display the correct frame that contains the needle and localize surgical tools in real time and with a good accuracy in water, polyvinyl alcohol cryogel phantom and liver tissue. Refresh rates of displaying the result is less than one second for volumes of size $67 \times 128 \times 224$ voxels. The time required to acquire this volume is about one second with the 4DC7-3/40 3D probe. So our application works at real-time.
\end{abstract}

Index Terms - 3D Ultrasound, needle localization, RANSAC, real-time application

\section{INTRODUCTION}

To reduce the invasive nature of many surgical operations like breast or liver biopsy, the use of thin tubular micro-tools is growing (most often they are needles and electrodes of diameter $1 \mathrm{~mm}$ and less). A precise navigation of such surgical instruments is essential on one hand for reducing the damage of biological tissue caused by failed insertions and on the other hand, for reducing the rate of sampling of a healthy tissue in a diseased organ. Ultrasound (US) imaging is used in clinics for its real-time monitoring and localization of such surgical tools and more other US is no-ionizing radiation (such as with X-rays) and it has relatively low costs compared with others imaging modalities.

In US images, the strong speckle noise and other acoustic artifacts make the appearance of a metallic tool irregular. So, even though doctors are trained for visual localization of such micro-tools in 2D US images, their observation remains a difficult task for them.

In this paper, we propose to assist the surgeon by providing a real-time application that automatically localizes the microtool and displays the section plane containing the tool from 3D US acquisitions. Variety of algorithms for object localization in ultrasound data were proposed, an overview can be found in [1] or in [2]. In this article, the axis of the micro-tool is estimated with a method based on model fitting using a Random Sample Consensus (RANSAC). This work aims at demonstrating the real time feasibility of the method.

\section{METHOD}

The algorithm used to localize the surgical tool from $3 \mathrm{D}$ data is presented in sub-section 2.1. The design of real-time application, overall functioning and implementation in $\mathrm{C}++$ is given in sub-section 2.2 which is a contribution of this paper.

\subsection{Localization algorithm}

The RANSAC procedure has been proposed by Fischler and Bolles [3] for robust estimation of model's parameters. Uherčík et al. [2] used it to determine the tool's axis of a curvilinear tool in a 3D ultrasound images with the following assumptions:

a) The axis of the tool is approximated by a curvilinear axis.

b) The length of the tool is larger than its diameter.

c) The tool is more echogenic than the background so it appears brighter.

Intensity of voxels belonging to the tool are more important than the biological tissue but some voxels from background also appear as high intensity. Therefore we use the model fitting with RANSAC procedure to estimate the location of the tool and eliminate those outliers. The proposed model involves several steps:

1. Pre-segmentation is done by thresholding in order to keep only high intensity voxels (assumption c)). The tentative voxels are selected and therefore the computing time is reduced.

2. Tool's axis is estimated using RANSAC. In the general case, it is represented by a spatial parametric polynomial curve of order 3 . However, in this application, we use only a straight line (assumption a)).

3. Local optimization is performed in order to obtain an accurate location of the tool. For real time purposes, the local optimization is not used here.

More detailed information can be found in [2, 4]. 


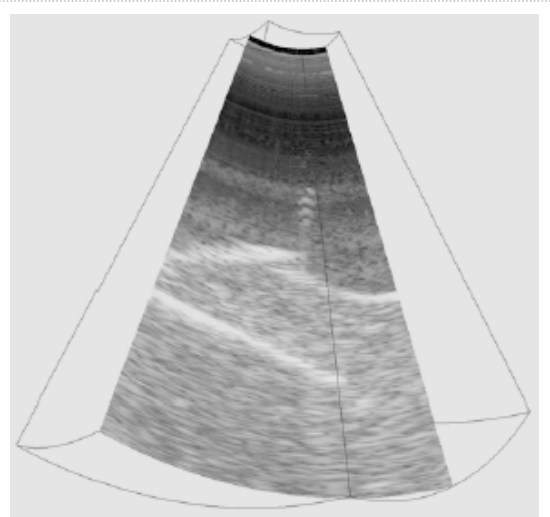

Figure 1: 3D view of a section plane containing a surgical tool in a 3D volume. Solid lines represent the boundaries of the acquired volume. The needle is clearly visible in this section plane.

This method has already been tested offline and has been validated in different configurations as described in [2]. From resulting tool's axis found by the above mentioned method and from the acquired volume geometry, the equation of the accurate plane containing the axis of the surgical tool is calculated (Figure 1). Note that this plane is not an acquired frame but is built from the US line including pixels of the tool.

\subsection{Proposed application}

The application is implemented on the Ultrasonix Sonix RP with a CPU speed of $3 \mathrm{GHz}$ and the Microsoft Windows XP operating system. The $3 \mathrm{D}$ probe $4 \mathrm{DC} 7-3 / 40$ that allows the acquisition of echographic volumes by built-in motorized sweeping of transducers was used. With this probe three frequencies are available for the acquisition of B-mode: 2.5 , 4.0 and $5.0 \mathrm{MHz}$. The array length is $55 \mathrm{~mm}$ and the maximum Field of View (FOV) is $104^{\circ}$.

The demonstration application is built from the open source application Propello provided by Ultrasonix ${ }^{1}$ for custom US applications. It uses the Porta library (version 4.6.2) which is an Application Programming Interface (API) for communication with the Ultrasonix scanner and for controlling the 3D probe motor. Propello was built-up in $\mathrm{C}++$ language using the Microsoft Foundation Class (MFC 7.1). This library allows, among others, multithreading which is used here to create a real-time application. The program source code was provided for the Visual Studio .NET environment.

The tool localization algorithm (model fitting by RANSAC procedure) was implemented in $\mathrm{C}++$ and it is provided as an open-source static library under GNU GPL licence ${ }^{2}$. The GNU Scientific library (GSL) version 1.11, an open-source library for scientific computing, was used for mathematical functions, e.g. matrix operations. In addition to the method [2], there is also a function for automatic estimation of the threshold. There are some important parameters of the tool

\footnotetext{
${ }^{1}$ Vancouver, BC, Canada

${ }^{2}$ http://www.creatis.insa-lyon.fr/site/en/ToolDemo
}

localization: minimal length of the tool, maximum radius of the cylinder, desired probability of the solution and maximal number of iterations.

\subsubsection{Functions and features}

Propello allows the acquisition of frame data from motorized 3D ultrasound transducers. Different types of data can be collected (pre-scan and post-scan converted B-mode data, unfiltered RF data, etc.). With this program, users have full control over the motor: there are two types of capture method: manual (i.e. step by step) and automatic mode which was used here.

After initialization, the user can modify various parameters for the acquisition, e.g. the number of frames per volume, degrees between each frame. After starting the automatic acquisition, all the acquired frames are stored in a cyclic buffer of a fixed size (Figure 2) according to the principle first-in, first-out (FIFO).

With the original application provided by Ultrasonix, it is not possible to know when an entire volume is acquired. We implemented this feature by testing the number of frames already acquired. Once a full volume has been obtained, it is ready to be processed by the tool localization (see also a detailed description of the procedure in the next section 2.2.2).

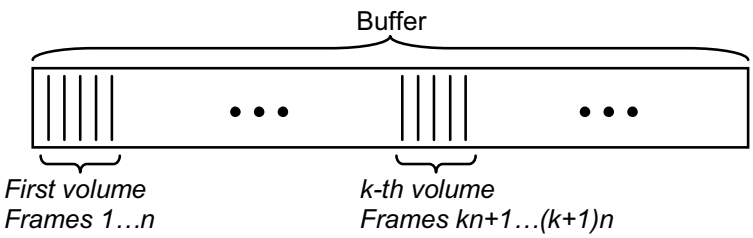

Figure 2: Representation of the cyclic buffer which contains acquired volumes.

An offline mode has also been implemented and is accessible from the graphical user interface (Figure 3). In this mode, user can replay entirely the acquired sequence and the tool localization either from the memory (i.e. the last acquired sequence in the scanner memory) or from a file which contains a sequence of volumes acquired previously. This feature (replay from file) allows the application to run on any computer without the Ultrasonix computer and without 3D probe connected.

\subsubsection{Software architecture}

We use multi-threading architecture, which allows running three different tasks in parallel:

- Main Window - for user interaction and displaying of the result of localization.

- Acquisition - for automatic acquisition of frames in Porta library.

- Worker Thread - for tool localization (using our $\mathrm{C}++$ library).

The synchronisation between them is done by calling functions and exchanging messages. The acquisition thread consists in checking if a correct probe (i.e. 3D probe) is connected; automatically moving the motor using parameters (e.g. spacing between frames and number of frames) supplied 
by the user, acquiring and storing frames in the cyclic buffer as discussed above.

Whenever a new frame is acquired, a callback function from Porta is called and handled in the main dialog (step 1). Once a full volume has been obtained (step 2), the software test if the worker thread has finished the processing of the previous volume (step 3). If finished, the main window wakes up the worker thread (step 4) which works on a copy of the last acquired volume. By this, the volume data is not overwritten by the acquisition thread. After the worker thread has computed the solution, it returns it to the main window for visualization (step 5). This acquisition runs in loop until the user stops it.

\subsubsection{Graphical user interface}

The Graphical User Interface (GUI) shows both the section plane containing the tool (marked by the number 2 in Figure 3) and the perpendicular plane to it (marked by the number 3 in Figure 3) in order to give a view of surrounding tissue. The section plane (containing the tool) is computed from the coordinates of the tool and from the geometry of 3D volume, so that the $2 \mathrm{D}$ slice completely contains the volume and the incident tool. The nearest neighbour interpolation technique is used to compute the image of the section plane with the tool. The perpendicular plane which intersects the middle of the tool is computed similarly. So, this application provides a relevant visualization (two perpendicular planes) for a realtime localization (Figure 3).

\section{RESULTS}

Experiments were performed with surgical tools submerged in water, inserted into homogeneous polyvinyl alcohol (PVA) Cryogel phantom [5] and a piece of beef liver. Conditions under which our experiments were performed are described in sub-section 3.1. The time required for tool localization and the success rate of localization are discussed in sub-section 3.2.

\subsection{Details of experiments}

For these experiments, the B-mode angle was $52^{\circ}$. The motor step was set to $0.732^{\circ}$ with a corresponding $49^{\circ} \mathrm{FOV}$ (i.e. 67 frames per volume). The maximum depth of acquisition was $6 \mathrm{~cm}$. The focal point was approximately at the depth of the tool. 3D volumes of size $33 \times 64 \times 224$ voxels have been acquired using the probe with central frequency 5 $\mathrm{MHz}$.

Concerning the material used in experiments, we used two types of needles: 1) Short injection needle of a length of $5 \mathrm{~cm}$ and a diameter of $1 \mathrm{~mm}$. 2) Longer biopsy needle (with a plastic covering) of length $6 \mathrm{~cm}$ and diameter $1.25 \mathrm{~mm}$. The PVA cryogel phantom of size approximately $5 \mathrm{~cm} \times 5 \mathrm{~cm} \times 5$ $\mathrm{cm}$ was used as mimicking tissue for insertion of the needles. The distance between the $3 \mathrm{D}$ probe and the needle was approximately $4 \mathrm{~cm}$.

First, our application was evaluated in static configuration, i.e. the needle was already inserted and was not moving. Second, the monitoring of the needle during its insertion inside the phantom was tested. Experiments were also performed with the biopsy needle in the liver tissue.
In the experiments, the following parameters have been set for tool localization: minimum length of the tool: $5 \mathrm{~mm}$; Maximum radius of the tool: $1 \mathrm{~mm}$; Maximum number of points (for automatic threshold estimation): 1000; Desired probability: 99.5\%; Maximum number of iterations: 3000 .

\subsection{Localization time and success rate}

In static configuration the application displays the correct frame containing the needle and gives an estimation of the needle position with a very high successful rate (higher than $98 \%$ ). When the needle is moving, in any environment this rate is decreasing slightly. Sometimes, although the displayed frame seems correct, the location of the needle is incorrect. On average, good frames are displayed with a success rate of about $95 \%$ and good locations of the needle are obtained with a success rate of about $75 \%$.

With a needle of diameter of $1 \mathrm{~mm}$, the mean time for processing of volumes (by tool localization algorithm) is about one second, i.e. the same order as the acquisition time of a $3 \mathrm{D}$ volume acquisition, so we can state that the application is a real time application. The localization time depends on the type of environment, varies linearly with the volume size and the number of points remaining after thresholding (a parameter of tool localization). For our setting, the time of processing is mostly less than the acquisition time of a volume.

For volumes whose size is smaller or equal to 67 planes $\mathrm{x}$ 128 beams x 224 samples (corresponding to a depth of $7 \mathrm{~cm}$, a field of view of $104^{\circ}$ in horizontal direction and $63^{\circ}$ in vertical direction), the acquisition time of a volume is about 1 second and the tool localization takes 0.98 second on average.

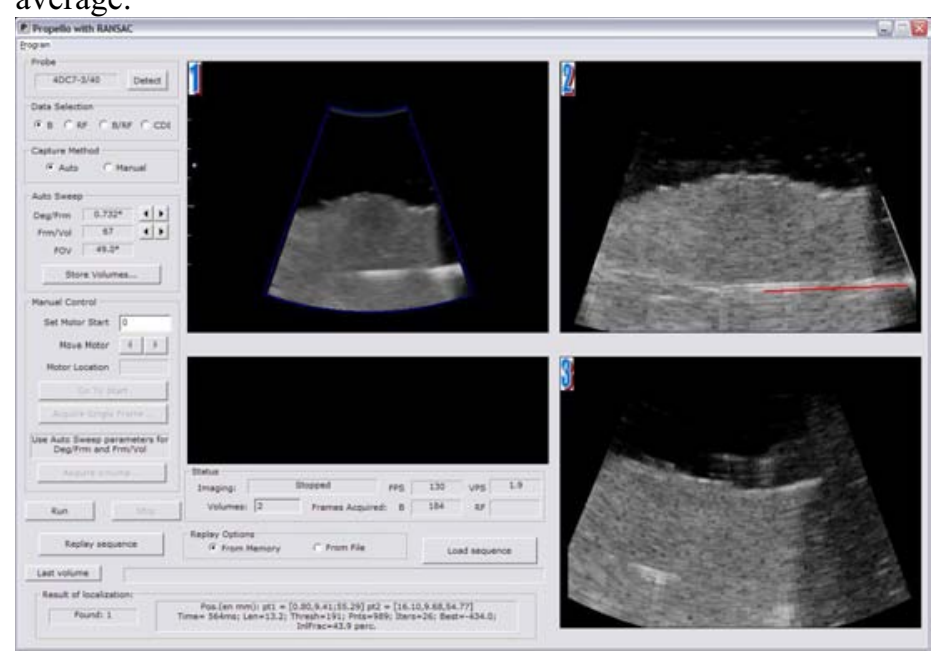

Figure 3: Modified user interface installed on Ultrasonix RP. The axis of the surgical tool is indicated by the red line. Several parameters can be changed from this window and the user can replay the acquisition sequence and tool localization either from the memory or from a file. 1) B-mode image. 2) Section plane. 3)

\section{DISCUSSION}

Seeing that it is feasible to localize the tool during its positioning in real time in various types of environment, the application can be useful for surgical operations after an optimisation phase of the process. In fact, because of images 
with low contrast, attenuation and artifacts, the automatic needle localization may be difficult.

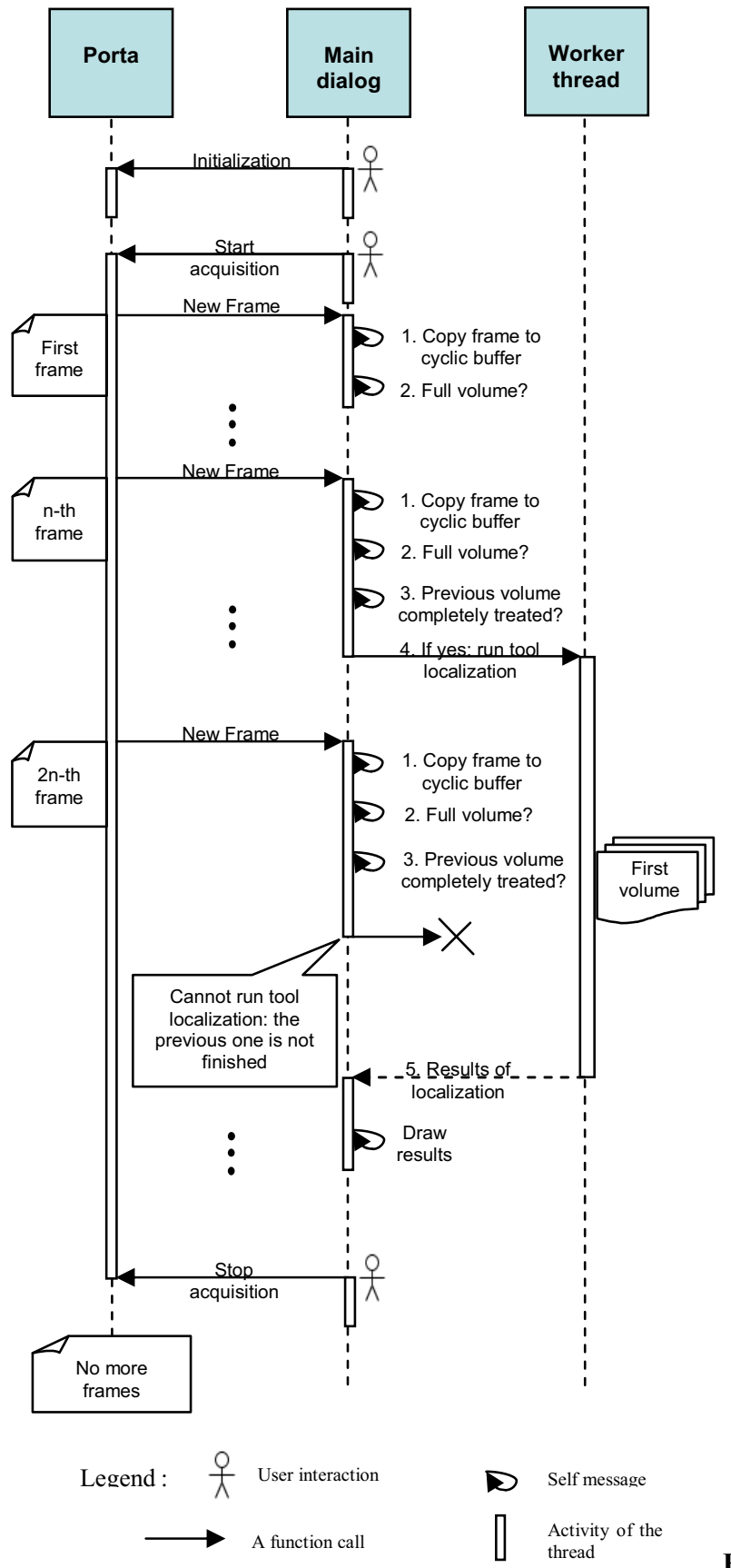

Figure

4: This figure shows how the synchronisation between Porta library, the main dialog and worker thread is done. Here, the user clicks on the initialize button, then run button and stop button which control the acquisition. Note that here a self message represents one method calling another method belonging to the same object. More explanations about this sequence diagram are given in sub-section 2.2.1.
It would be interesting to implement also the other tool models based on the intensity profile of the tool as proposed in [2]. To enhance the robustness of localization, using specific method of ultrasound acquisition like pulse inversion could improve the tool/background contrast. In [6] the authors propose the use of line filtering method to exploit the onedimensionality of the tool to overcome these problems.

The resulting location of the tool varies much. This is because the tool localization is done separately on each volume from the sequence. The information computed from the previous volume could be used to constrain the estimation for the tracking of the tool in sequence of volumes.

\section{CONCLUSION}

We propose to assist surgeons by providing a real-time application which is able to track surgical tools movements in real time. The refresh rate of images which give the two perpendicular planes (one containing the axis of the surgical tool and the other perpendicular to it) is about one second. It corresponds to the time necessary to acquire a full volume with the 3D probe used here. In the absence of artifacts, our experiments have shown that we are able to locate the surgical tool in water, PVA cryogel phantom and liver quickly and with a good accuracy. The application is available at our webpage for download.

Perpendicular plane.

\section{ACKNOWLEDGMENTS}

The author Marián Uherčík was supported by an EC project MEST-CT-2005-021024 WARTHE.

\section{REFERENCES}

[1] M. Barva, M. Uherčík., J.-M. Mari, J. Kybic, J.-R. Duhamel, H. Liebgott, V. Hlavac, C. Cachard. Parallel integral projection transform for straight electrode localization in 3-D ultrasound images. IEEE Trans. on Ultrasonics, Ferroelectrics, and Frequency Control, 2008. 55(7): p. 1559-1569.

[2] M. Uherčík, J. Kybic., H. Liebgott, C. Cachard. Model Fitting using RANSAC for Surgical Tool Localization in 3D Ultrasound Images. IEEE Trans. on Biomed. Eng., 58(8): pp. 1907-1916, 2010.

[3] M.A. Fischler and R.C. Bolles. Random sample consensus: a paradigm for model fitting with applications to image analysis and automated cartography. Communications of the ACM, 1981. 24(6): p. 381-395.

[4] M. Barva, J. Kybic, J.-M Mari, C. Cachard, V. Hlavac. Localizing metal electrode from $3 D$ ultrasound data using RANSAC and intensity priors. In IFMBE Proceedings EMBEC' 2005.

[5] F. Duboeuf, A. Basarab, H. Liebgott, E. Brusseau, P. Delachartre, D. Vray. Investigation of PVA cryogel Young's modulus stability with time, controlled by a simple reliable technique. Medical Physics, 2009. 36(2): p. 656-661.

[6] M. Uherčík, J. Kybic, C. Cachard, H. Liebgott. Line filtering for detection of microtools in 3D ultrasound data, 2009. In Proceedings of IEEE International Ultrasound Symposium 2009, Rome, Italy. 